\title{
Subcutaneous Calcaneal Bursitis - A Commonly Forgotten Differential Diagnosis
}

\section{Pedro Henrique de Oliveira Alvarenga ${ }^{1}$, Mayara Oliveira da Silva ${ }^{2}$, Márcio Luís Duarte ${ }^{3,4 *}$ and Lucas Ribeiro dos Santos ${ }^{4,5}$}

${ }^{1}$ Biomedicine Department, Universidade São Judas, Santos, São Paulo, Brazil

${ }^{2}$ Biomedicine Department, Universidade Paulista, Santos, São Paulo, Brazil

${ }^{3}$ Radiology Department, Centro Radiológico e Especialidades Médicas São Gabriel,

Praia Grande, São Paulo, Brazil

${ }^{4}$ Master Degree in Evidence-Based Health at UNIFESP, São Paulo, São Paulo, Brazil

${ }^{5}$ Professor of Physiology, Faculdade de Ciências Médicas de Santos, Santos, São Paulo,

Brazil

*Corresponding Author: Márcio Luís Duarte, Radiology Department, Centro

Radiológico e Especialidades Médicas São Gabriel, Praia Grande, São Paulo, Brazil.
Received: June 09, 2021

Published: June 30, 2021

(C) All rights are reserved by Márcio Luís

Duarte., et al.

\section{Abstract}

The bursa is a fluid-filled synovial bag that can be profound or on the surface and serves as a pad to diminish friction among structures like tendons, bone, or skin. The bursa positioned in the subcutaneous tissue between the bone and the skin nearby is the superficial bursa. Adventitious or non-native bursa develops secondary to chronic microtrauma and shearing forces exerted on the subcutaneous tissue. We report a 40 years-old woman who complains about pain in the posterior region of the ankle for four months. A physical examination refers to pain on palpation of the posterior region of the ankle, and with the dorsiflexion of the ankle, but without limitation of movement. It presents slight edema in the region without alteration of the skin color. At ultrasonography she presents fluid in the subcutaneous calcaneal bursa with a normal calcaneus tendon, characterizing subcutaneous calcaneal bursitis.

Keywords: Calcaneus; Ankle Joint; Ultrasonography

\section{Introduction}

The feet transfer the weight of the body to the floor and keep the stability of posture through movement [1]. When the foot hit, the feet work as a shock absorber and then shift the lever for pushoff [1]. Considering that the weight of the entire body is supported by the feet, the appropriate muscles, tendons, or ligaments are predisposed to damage [1]. Foot disturbances limit walking and worse the quality of life of the patient [1].

The bursa is a fluid-filled synovial bag that can be profound or on the surface and serves as a pad to diminish friction among structures like tendons, bone, or skin [2,3]. The bursa positioned in the subcutaneous tissue between the bone and the skin nearby is the superficial bursa [2]. Adventitious or non-native bursa develops secondary to chronic microtrauma and shearing forces exerted on the subcutaneous tissue $[2,4]$.

\section{Case Report}

A 40 years-old woman complain about pain in the posterior region of the ankle for four months. Refers worsening when walking and wearing tight shoes. Denies previous surgeries, diseases and sports activities. Physical examination refers to pain on palpation of the posterior region of the ankle and with the dorsiflexion of the ankle, but without limitation of movement. It presents slight edema in the region without bruises. 
An ultrasonography using a linear high-frequency probe (4 - 14 $\mathrm{MHz}$ ) demonstrated fluid in the subcutaneous calcaneal bursa with a normal calcaneus tendon (Figure 1 and 2), characterizing subcutaneous calcaneal bursitis. The patient underwent treatment with an "ice pack" - cryotherapy - at the site for 10 days, reporting improvement without the need for drug treatment.

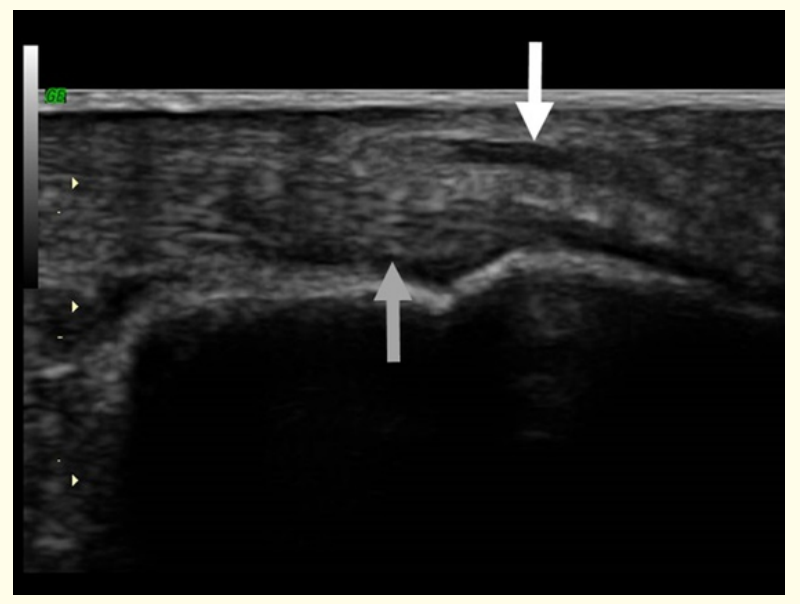

Figure 1: Ultrasonography demonstrating fluid in the subcutaneous calcaneal bursa (white arrow) with a normal calcaneus tendon (gray arrow).

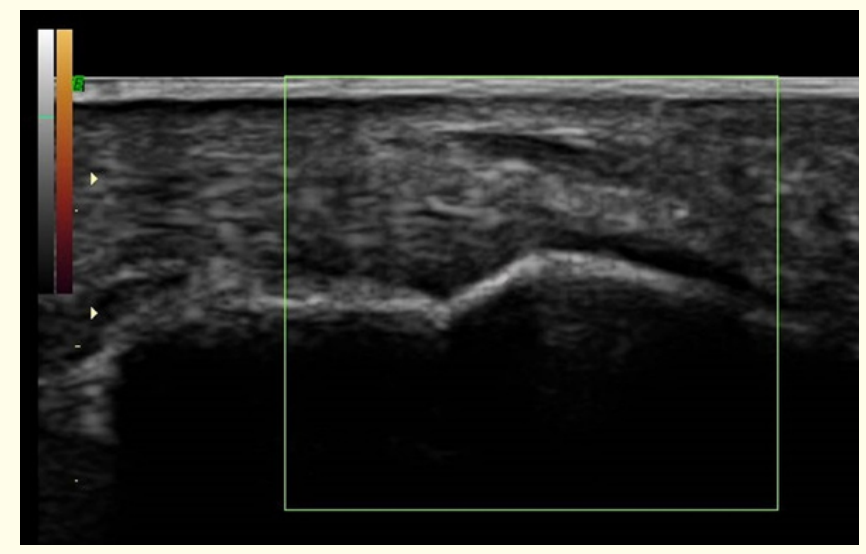

Figure 2: Ultrasonography demonstrating fluid in the subcutaneous calcaneal bursa without vascularization on Doppler study.

\section{Discussion}

The subcutaneous calcaneus bursa, also known as Achilles bursa, is superficially located amidst the Achilles tendon and the neighboring subcutaneous tissue [2,3]. It's an adventitious bursa and the presence of fluid and it is always considered abnormal $[2,3]$. Subcutaneous calcaneal bursitis is an unusual infirmity essentially generated by Haglund deformity or using inadequate footwear (e.g. too tight) [2]. High heels are also a major risk factor [2]. The disease happens in a higher frequency in skaters, dancers, and rowers and its most common pathophysiologic mechanism is chronic microtrauma [2].

Chronic superficial bursitis usually has edema around the affected bursa, but frequently generates minimum pain or is painless [2]. Cases of acute traumatic/hemorrhagic bursitis or septic bursitis may have meaningful pain, sensibility, and reduced mobility of the region [2]. The subcutaneous bursa has a higher level of innervation when compared to other ankle bursae and may correspond to a clinically important cause of pain [5].

There is a large differential diagnosis for superficial bursitis [2]. It includes infection (septic bursitis), cellulitis, septic and/or inflammatory arthritis, joint effusions and Morel-Lavallée injury [2]. Image tests can aid to diagnose subcutaneous calcaneus bursitis.

In cases of trauma with suspicion for fracture, a foreign body, calcification, or bony abnormality, like Haglund deformity, plain radiography can help [2]. To estimate the area and size of the foot lesions, ultrasonography can be utilized because it provides realtime imaging and dynamic evaluation [1]. It can analyze a large bursa when important soft-tissue edema is originated by cellulitis the soft-tissue edema limits the physical examination to determine whether a bursa is involved [2]. Static and dynamic evaluation with ultrasonography are essential for differential diagnosis [1]. An important detail for the sonographer is as the bursa is superficial, it requires an ultrasound scanning with minimum transducer pressure and a thick layer of gel [3].

The inflammatory process (hyperemia) can be visualized by the color Doppler ultrasonography. ${ }^{2}$ When the color Doppler is negative it reduces heavily the probability of inflammatory bursitis (septic or aseptic). ${ }^{2}$ Magnetic resonance imaging (MRI) should be 
performed if there is a suspicion of septic bursitis and aspiration of the bursa is unsuccessful [2]. The enhancement in MRI cannot differentiate among the inflammatory changes of septic and aseptic bursitis [2]. But the lack of enhancement means that septic bursitis is not present [2].

The majority of patients with microtraumatic superficial bursitis have a satisfactory outcome to conservative treatment with the elevation of the limb, compression wraps, movement adjustment, ice, and analgesics [2]. In patients with acute hemorrhagic bursitis with significant bursal enlargement which interferes with daily activities, the bursal aspiration may reduce the extent of symptoms [2].

\section{Conclusion}

We report a case of subcutaneous calcaneus bursitis, an unusual lesion, often unsuspected in the initial clinical evaluation, but which can simulate more complex lesions. Although it is a disease commonly overlooked, it has an easy and efficient treatment.

\section{Conflict of Interest}

The authors declare that there is no conflict of interests regarding the publication of this paper.

\section{Patient Consent}

The written informed consent of the patient was obtained, for the publication of her case.

\section{Bibliography}

1. Chang MC., et al. "A Rare Case of Lateral Forefoot Pain: Plantar Adventitious Bursitis". Cureus 12.7 (2020): e9011.

2. Khodaee M. "Common Superficial Bursitis". American Family Physician 95.4 (2017): 224-231.

3. Ruangchaijatuporn T., et al. "Ultrasound evaluation of bursae: anatomy and pathological appearances". Skeletal Radiology 46.4 (2017): 445-462.

4. Zidani H., et al. "Adventitious bursitis in the plantar fat pad of forefoot presenting as a tumoral mass". Journal of Radiology Case Reports 14.2 (2020): 12-20.

5. Lui TH., et al. "Minimally Invasive and Endoscopic Treatment of Haglund Syndrome". Foot and Ankle Clinics 24.3 (2019): 515-531.
Volume 4 Issue 7 July 2021

(C) All rights are reserved by Márcio Luís Duarte., et al. 